Proceedings of the 42th "Jaszowiec" International School and Conference on the Physics of Semiconductors, Wisła 2013

\title{
Optical Properties of Molybdenum Disulfide $\left(\mathrm{MoS}_{2}\right)$
}

\author{
K. Golasa, M. Grzeszczyk, K.P. Korona, R. Bożek, J. Binder, J. Szczytko, \\ A. WYSMOŁEK AND A. BABIŃsKi
}

Institute of Experimental Physics, Faculty of Physics, University of Warsaw, Hoża 69, 00-681 Warsaw, Poland

\begin{abstract}
Research of a monolayer and a bulk $\mathrm{MoS}_{2}$ is reported. The room temperature Raman spectra of the natural $\mathrm{MoS}_{2}$ crystals for the both resonant $(632.8 \mathrm{~nm})$ and the non-resonant $(532 \mathrm{~nm})$ excitation are presented. The apparent differences observed in the spectra from the bulk and the one monolayer $\mathrm{MoS}_{2}$ are discussed. In particular, the feature due to a first order scattering involving the LA $(M)$ phonon in the resonance Raman spectrum of the one monolayer $\mathrm{MoS}_{2}$ was observed and explained in terms of the disorder in the natural crystal. The disorder is also documented by the line-shape of the room-temperature photoluminescence spectra observed from both the bulk and the one monolayer $\mathrm{MoS}_{2}$.
\end{abstract}

DOI: $10.12693 /$ APhysPolA.124.849

PACS: 63.22.-m, 71.35.-y, 78.20.-e, 78.30.-j

\section{Introduction}

Layered transition metal dichalcogenide materials (LMDCs) in which layers of covalently bound atoms are weakly bound by van der Waals forces have been recently objects of intense studies [1]. It is related to their properties which substantially change as a result of the transition from their 3D to 2D form. Molybdenum disulfide $\left(\mathrm{MoS}_{2}\right)$ is a typical representative of the LMDCs family [2]. It was suggested that a $\mathrm{MoS}_{2}$ monolayer could complement graphene in applications, which require thin and transparent semiconductors, such as optoelectronics and energy harvesting. This is because contrary to graphene, $\mathrm{MoS}_{2}$ is a semiconductor of a non-zero energy gap. This makes the investigation of basic properties of a few-layered $\mathrm{MoS}_{2}$ of importance for development of the semiconductor materials' science. Although properties of bulk $\mathrm{MoS}_{2}$ were thoroughly investigated both theoretically and experimentally [3], less information is available on extended two-dimensional $\mathrm{MoS}_{2}$ sheets, down to unit cell thickness, in which quantum confinement effects become important.

The research reported in this communication aims at broadening the knowledge on the properties of a $\mathrm{MoS}_{2}$ monolayer. We studied the optical properties of a monolayer (1 ML) $\mathrm{MoS}_{2}$ using the Raman spectroscopy and the photoluminescence (PL) measurements and compare them with the results obtained on a bulk crystal.

\section{Experimental procedure}

The samples of $\mathrm{MoS}_{2}$ studied in this work were obtained using a mechanical exfoliation of a naturally occurring molybdenum ore - molybdenite from the Molly Hill deposit in Canada. The method has been used since the 1960s [4] to prepare and study the properties of a few-layered LMDCs, such as $\mathrm{MoS}_{2}$ and $\mathrm{NbSe}_{2}$ and by Geim and Novoselov in their studies of graphene [5]. The mechanical exfoliation is considerably less destructive than other methods and has successfully been used to create large, $10 \mu \mathrm{m}$ single-layer flakes on a variety of substrates [1]. The exfoliated $\mathrm{MoS}_{2}$ samples were deposited on the dielectric-coated $\mathrm{SiO}_{2} / \mathrm{Si}$ substrates, which helped to visualize and locate single- and few-layered flakes. The color of the dielectric-coated substrate depends on an interference effect from the reflection from the two surfaces of the dielectric layers. Single- and few-layered flakes on the surface of the dielectric modify the interference, as a result a color contrast between the flake and the substrate can be observed [6].

Optical microscopy measurements were performed with a resonant $(\lambda=632.8 \mathrm{~nm})$ and a non-resonant excitation $(\lambda=532 \mathrm{~nm})$ using a T64000 Series II Raman spectrometer. The spectrometer has both the modes: single- and triple-grating, which allows to get to a distance of $20 \mathrm{~cm}^{-1}$ to the laser line.

\section{Results and discussion}

The Raman spectroscopy is a non-destructive characterization method for studying the electronic and vibrational properties in materials. The main features of the Raman spectrum of the bulk $\mathrm{MoS}_{2}$ excited non-resonantly are four modes, namely, $E_{2 \mathrm{~g}}^{2}$ (in-plane optical vibration of the rigid atomic bond), $E_{1 \mathrm{~g}}$ (in-plane optical vibration of $\mathrm{S}$ atoms in the basal plane), $E_{2 \mathrm{~g}}^{1}$ (in-plane optical vibration of the $\mathrm{Mo}+\mathrm{S}$ atoms in the basal plane) and $A_{1 \mathrm{~g}}$ (out-of-plane optical vibration of S atoms along the $c$ axis) [7]. The spectrum substantially changes as the thickness of the sample reaches the 1 ML limit. Ultimately, in the $1 \mathrm{ML} \mathrm{MoS}_{2}$ the frequency difference between the two Raman active modes near $400 \mathrm{~cm}^{-1}$ namely $E_{2 \mathrm{~g}}^{1}$ and $A_{1 \mathrm{~g}}$ equals $18.4 \mathrm{~cm}^{-1}$ (see Fig. 1). This observation usually serves as a fingerprint of the $1 \mathrm{ML}$ thickness of a sample [8].

Let us focus first on the Raman spectra excited non-resonantly (see Fig. 2). The main difference between the spectra from the bulk and the $1 \mathrm{ML} \mathrm{MoS}_{2}$ is related to the substantial decrease of the signal intensity for the $1 \mathrm{ML}$ 


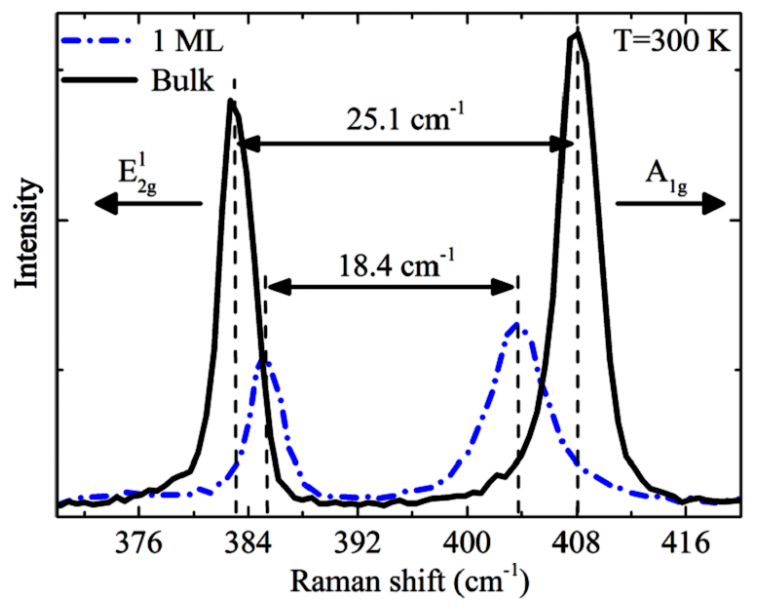

Fig. 1. Raman active modes in the investigated bulk material and the $1 \mathrm{ML} \mathrm{MoS}_{2}$.

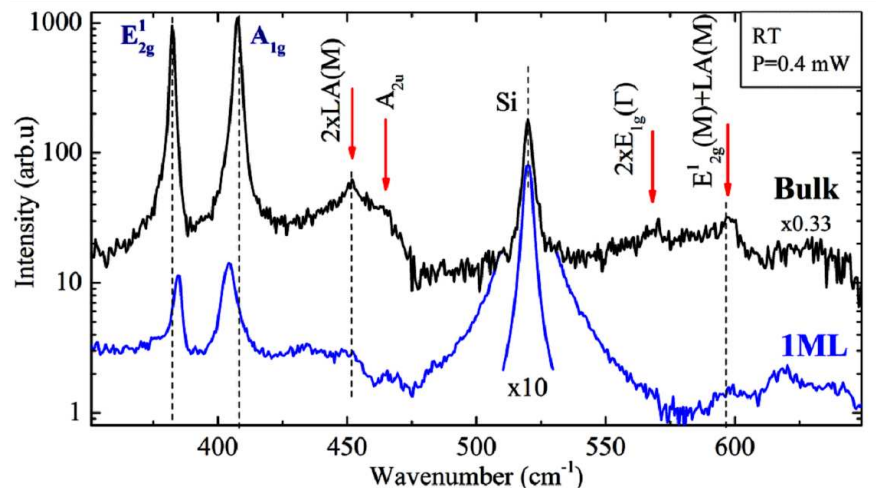

Fig. 2. Non-resonance room-temperature Raman scattering spectrum from the bulk and the $1 \mathrm{ML} \mathrm{MoS}_{2}$.

structure and the previously mentioned change in the energy difference between the basic Raman-active modes $E_{2 \mathrm{~g}}^{1}$ and $A_{1 \mathrm{~g}}$.

The spectrum obtained with the resonant excitation is richer. In resonance Raman spectroscopy, the frequency of the laser beam is adjusted to be near to an electronic transition (resonance). It leads to a significant increase of the Raman scattering intensity of the vibrational modes, which particular electronic transition is associated with. Simultaneously, the Raman signals from the other transitions are engulfed. Whereas the non-resonance Raman spectrum is dominated by the two modes due to the zone-center first-order Raman scattering [9], the resonance Raman spectrum of the bulk $\mathrm{MoS}_{2}$ consists of more peaks (see Fig. 3), which are enhanced by an interaction with excitonic electron transitions [10, 11]. In this report we focus our attention on the following features. The most intensive peak, asymmetric and centered at $\approx 460 \mathrm{~cm}^{-1}$ was originally assigned to the second order $2 \times \mathrm{LA}(M)$ mode [10]. Frey et al. proposed later that the peak comprises two components, one of which is $2 \times \operatorname{LA}(M)[12]$. The attribution of the other peak is not yet clear. In the higher frequency region there are peaks observed at 572,599 , and $641 \mathrm{~cm}^{-1}$, which are assigned following Ref. [10] to the second-order processes: $2 \times E_{1 \mathrm{~g}}(\Gamma), E_{2 \mathrm{~g}}^{1}(M)+\mathrm{LA}(M)$, and $A_{1 \mathrm{~g}}(M)+\mathrm{LA}(M)$, respectively. The non-resonance Raman spectrum from the $1 \mathrm{ML} \mathrm{MoS}_{2}$ significantly differs from that of the bulk material. Besides the change in the energy position of the $E_{2 \mathrm{~g}}^{1}$ and $A_{1 \mathrm{~g}}$ following changes are observed: the mode at $\approx 460 \mathrm{~cm}^{-1}$ gains in intensity and uncovers its bimodal character, the mode at $\approx 230 \mathrm{~cm}^{-1}$ emerges and the high energy $E_{2 \mathrm{~g}}^{1}(M)+\mathrm{LA}(M)$, and $A_{1 \mathrm{~g}}(M)+\mathrm{LA}(M)$ features become unresolved.

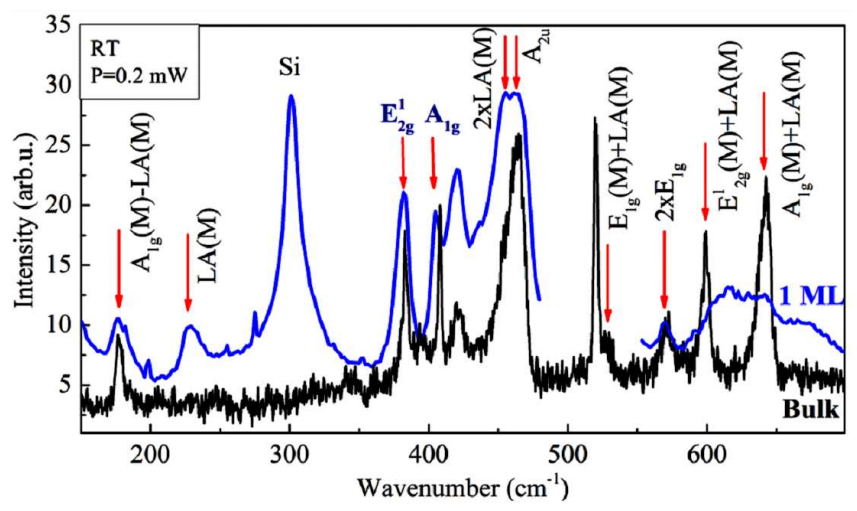

Fig. 3. Room-temperature Raman scattering spectrum from the bulk and the $1 \mathrm{ML} \mathrm{MoS}_{2}$ with the resonant $(632.8 \mathrm{~nm})$ excitation.

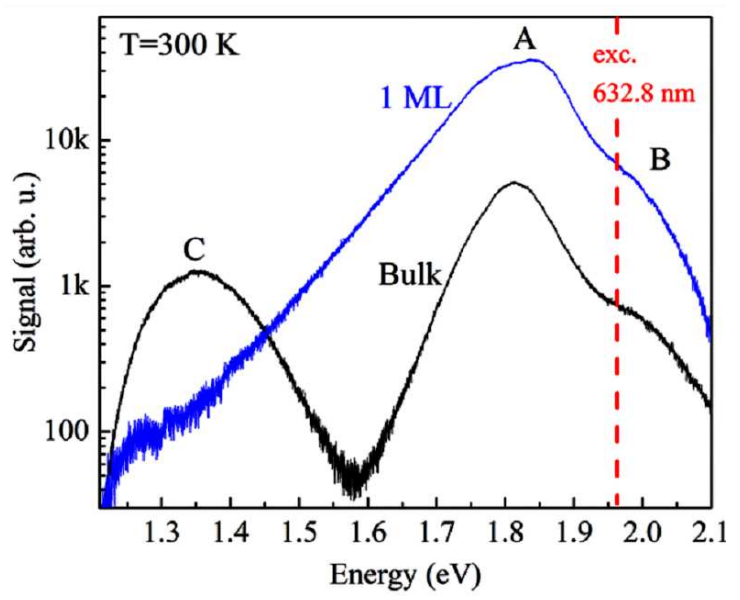

Fig. 4. Room-temperature photoluminescence spectra in the bulk material and the $1 \mathrm{ML} \mathrm{MoS}$. Measurements were performed with a non-resonant excitation $(\lambda=$ $532 \mathrm{~nm})$. The energy of the resonant excitation used for the Raman scattering measurements is shown for comparison.

Following the discussion presented in Ref. [10], we relate the appearance of the $\approx 230 \mathrm{~cm}^{-1}$ mode to the first order scattering of the $\mathrm{LA}(M)$ phonon allowed in the spectrum due to a disorder in the sample. The disorder 
allows also the optical transitions through the indirect energy gap, which in the $1 \mathrm{ML}$ sample approximately equals the excitation laser energy. The resonance may amplify the normally forbidden scattering even further. The effect of the disorder may also be responsible for the broadening of the high-energy modes which prevents their resolution.

The presence of the disorder in the investigated natural crystals was also confirmed by the results of the PL measurements (see Fig. 4). It can be seen that the PL intensity from the bulk is significantly weaker in comparison to the PL from the $1 \mathrm{ML}$ sample. This reflects the indirect character of the band-gap in the former material. The A and $\mathrm{B}$ transitions are related to the spin-orbit splitting in the valence band maximum, the peak $\mathrm{C}$ reflects the indirect band-gap transition in the bulk material [13]. The low-energy tail of the interband transition (A) is related to the disorder in the investigated material.

\section{Conclusions}

In conclusion, optical properties of the natural bulk and the one monolayer $\mathrm{MoS}_{2}$ crystals have been studied by means of the PL and the Raman spectroscopy. Significant difference between the Raman spectra for the resonant $(632.8 \mathrm{~nm})$ and the non-resonant $(532 \mathrm{~nm})$ excitations were observed for the both investigated structures. The feature due to a first order scattering of the $\mathrm{LA}(M)$ phonon observed in the resonance Raman spectrum of the one monolayer $\mathrm{MoS}_{2}$ was explained in terms of the disorder in the natural crystal. The disorder has also been documented by the line-shape of the room-temperature photoluminescence spectra observed from both the bulk and the one monolayer $\mathrm{MoS}_{2}$.

\section{Acknowledgments}

The authors acknowledge fruitful discussions with M. Potemski and P. Leszczyński from the Grenoble National Laboratory of High Magnetic Fields.

\section{References}

[1] S.Z. Butler, S.M. Hollen, L. Cao, Y. Cui, J. A. Gupta, H.R. Gutiérrez, T.F. Heinz, S.S. Hong, J. Huang, A.F. Ismach, E. Johnston-Halperin, M. Kuno, V.V. Plashnitsa, R.D. Robinson, R.S. Ruoff, S. Salahuddin, J. Shan, L. Shi, M.G. Spencer, M. Terrones, W. Windl, J.E. Goldberger, ACS Nano 7, 2898 (2013).

[2] X. Hunag, Z.Y. Zeng, H. Zhang, Chem. Soc. Rev. 42, 1934 (2013).

[3] J.A. Wilson, A.D. Yoffe, Adv. Phys. 18, 193 (1969).

[4] R.F. Frindt, J. Appl. Phys. 37, 1928 (1966).

[5] K.S. Novoselov, D. Jiang, F. Schedin, T.J. Booth, V.V. Khotkevich, S.V. Morozov, A.K. Geim, Proc. Natl. Acad. USA 102, 10451 (2005).

[6] P. Blake, E.W. Hill, A.H. Castro Neto, K.S. Novoselov, D. Jiang, R. Yang, T.J. Booth, A.K. Geim, Appl. Phys. Lett. 91, 063124 (2007).

[7] C.V. Ramana, U. Becker, V. Shutthanandan, C.M. Julien, Geochem. Trans. 9, 8 (2008).

[8] A. Molina-Sanchez, L. Wirtz, Phys. Rev. B 84, 155413 (2011).

[9] T.J. Wieting, J.L. Verbie, Phys. Rev. B 3, 4286 (1971).

[10] J.M. Chen, C.S. Wang, Solid State Commun. 14, 857 (1974).

[11] G.L. Frey, R. Tenne, M. Matthews, M.S. Dresselhaus, G. Dresselhaus, Phys. Rev. B 60, 2883 (1999).

[12] A.M. Stacy, D.T. Hodul, J. Phys. Chem. Solids 46, 405 (1985).

[13] K.F. Mak, C. Lee, J. Hone, J. Shan, T.F. Heinz, Phys. Rev. Lett. 105, 136805 (2010). 